\title{
Población, empleo y pobreza en los territorios rurales de Costa Rica
}

Recibido: 2 de abril 2018

Revisado: 2 de mayo 2018

Aprobado: 23 de mayo 2018

\section{Ronald Rivera Alfaro}

Costarricense. Doctor en

Ciencias Sociales por la

Universidad Nacional de

Costa Rica. Académico del

Instituto de Estudios del

Trabajo (IESTRA) de la

Universidad Nacional.

Correo electrónico:

ronaldrive@gmail.com

Ángel Jesús Porras Solís

Costarricense. Máster en

Técnicas Estadísticas por la

Universidad de Santiago de

Compostela (España).

Académico de la Escuela de

Ciencias Agrarias de la

Universidad Nacional.

Correo electrónico: angel.porras.solis@una.cr
Resumen: En el presente artículo se examinan las principales características de la población rural en Costa Rica a la luz de la Encuesta Nacional de Hogares (ENAHO 2017), prestando atención a variables socioeconómicas como la educación, el empleo, los ingresos la migración y la pobreza. Asimismo, se aporta una serie de elementos y reflexiones relacionados con el reposicionamiento de los territorios rurales en términos de la gobernanza y la acción ciudadana y la necesidad de llevar a cabo una estrategia para impulsar el empleo local y rural.

Palabras clave: Territorios rurales; empleo; ciudadanía; pobreza, desarrollo rural

\section{Population, Employment and Poverty in the Rural Territories of Costa Rica}

Abstract: This paper examines the main characteristics of the rural population in Costa Rica in light of the National Household Survey (ENAHO 2017), paying attention to socioeconomic variables such as education, employment, income, migration and poverty. It also provides a series of elements and reflections related to the repositioning of rural territories in terms of governance and citizen action and the need to carry out a strategy to boost local and rural employment.

Key words: Rural territory; employment; citizen; poverty, rural development

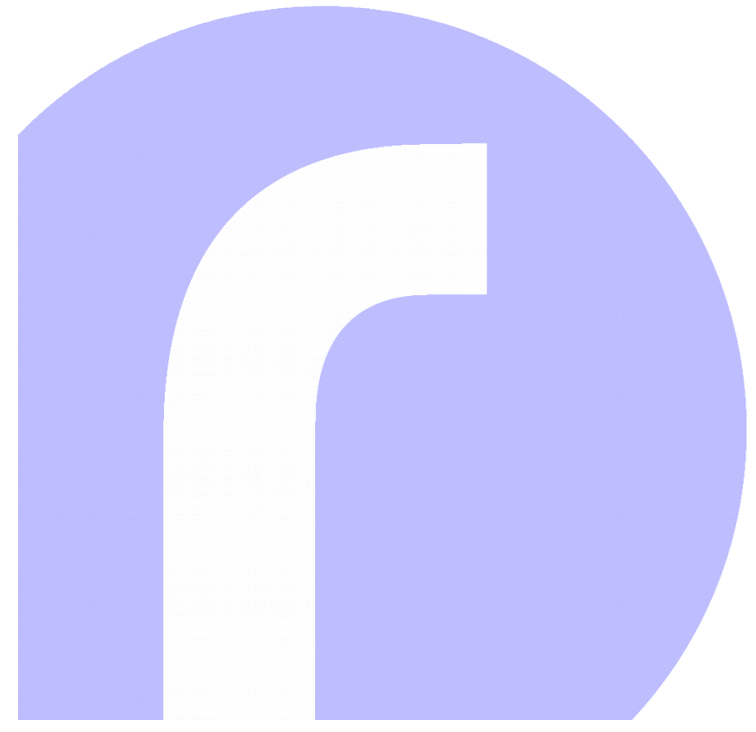




\section{Introducción}

La generación de empleos de calidad en territorios rurales es uno de los principales retos de los gobiernos latinoamericanos, ya que la concentración de la riqueza se presenta en los centros urbanizados e industrializados donde se aglomera el empleo formal y la institucionalidad gubernamental. Este desbalance estructural crea intensas transformaciones sociales en las dinámicas familiares y de elección educativa que, por hecho consustancial, legitiman tal desigualdad funcional del modelo de desarrollo capitalista.

En este sentido, la condición que determina la coexistencia de las posibilidades reales de tener un ingreso estable mediante un trabajo está limitada a la migración laboral, ya que las limitantes para la generación de empleo, la vinculación con los mercados y la dinamización de actividades productivas en los territorios rurales se mantienen al margen de sus particularidades ecosociales, paisajísticas y culturales; y, con ello, un amputamiento de los modos de producción diferenciados, incluidas la composición de la fuerza de trabajo y las relaciones sociales que se crean.

En el caso de Costa Rica, la cantidad de personas que habitan los territorios rurales entre 1960 al 2015 bajó en 43\%. Para el 2017, según la Encuesta Nacional de Hogares (ENAHO) del INEC, en la zona rural se estima que habita un total de 1.354.630 personas, correspondiente a 418.937 hogares. Esto significa, aproximadamente, $27 \%$ de la población y hogares totales del país. A su vez, cerca de $40 \%$ de la población se dedica a ocupaciones elementales (no calificadas), principalmente relacionadas con la agricultura; y $56 \%$ de los hogares se encuentran ubicados en los dos quintiles de ingresos más bajos, lo cual también implica que casi $25 \%$ de las personas viva en condición de pobreza.

A la luz de los datos que proporciona la ENAHO 2017, en el presente artículo se examinan las principales características de la población rural en Costa Rica. Se enfatizan aspectos relacionados con la composición de los hogares, la educación, el empleo, los ingresos, la migración y la pobreza. Hoy, la ENAHO se constituyen como una de las principales fuentes de información que se producen en el país y que muestran, de forma detallada, una radiografía anual de lo que sucede en los hogares costarricenses y por región de planificación, principalmente en términos de empleo, ingresos, pobreza y desigualdad.

En esa línea, el artículo aporta una serie de elementos y reflexiones a esta discusión, que van desde el reposicionamiento de los territorios rurales en términos de la gobernanza y la acción ciudadana, hasta la necesidad de ir trazando el rumbo económico, empleo y desarrollo rural, en un contexto marcado por la informalidad y atisbos de pobreza. Por último, se hace hincapié en la importancia y necesidad de llevar a cabo una estrategia para impulsar el empleo local y rural. 


\section{Aspectos metodológicos}

Se toma como fuente de información la Encuesta Nacional de Hogares 2017 (ENAHO 2017), considerando como unidad de análisis el hogar y los respectivos miembros que lo conforman. En tal sentido, el INEC (2017 (a), 14) define el hogar como "personas con vínculos familiares o sin ellos, que residen habitualmente en una vivienda individual, que participan de la formación, utilización, o ambas, de un mismo presupuesto, que llevan una vida en común, y elaboran y consumen en común sus alimentos".

Dado que los dominios de estudio "son subpoblaciones generalmente relacionadas a áreas geográficas para las cuales se requiere estimaciones con una precisión conocida, el diseño muestral de la ENAHO considera como dominios de estudio el nivel nacional, zona urbana y rural, y las seis regiones de planificación" (INEC 2017 (a), 22). La zona responde a la clasificación de "urbana" o "rural" que a partir de ciertos criterios se asignan a los segmentos censales de acuerdo con su grado de urbanización. Para los intereses del presente trabajo, el dominio de estudio se enmarca en la zona rural nacional y la zona rural de cada una de las seis regiones de planificación. La zona rural incluye aquellos segmentos clasificados con grado de urbanización rural concentrado y rural disperso.

El término rural concentrado reúne las siguientes características: el suelo está ocupado predominantemente por actividades no agropecuarias; hay 50 o más viviendas agrupadas o contiguas; en general, la distancia entre ellas no es mayor a los 20 metros; disponen de algún servicio de infraestructura como electricidad domiciliaria, agua potable o teléfono; y cuentan con algunos servicios como escuela, iglesia, centro de salud, puestos de salud y guardia rural. Por su parte, rural disperso hace referencia a todas las áreas que no pertenecen a alguna de las clasificaciones anteriores (INEC, 2001, 13)

La ENAHO 2017 considera una muestra total efectiva de 10.712 hogares. La cantidad de hogares rurales considerados para el presente trabajo es 3.332 $(31,1 \%)$, que corresponde a una población de 418.935 hogares y 1.354 .630 personas, una vez aplicados los factores de expansión respectivos.

\section{Características de la población y el empleo rural}

De acuerdo con la ENAHO 2017, se estima que en la zona rural habitan un total de 1.354 .630 personas, correspondiente a 418.935 hogares. Como se muestra en la tabla 1, la región Central concentra $31 \%$ de los hogares y personas de los territorios rurales; el restante $69 \%$ se distribuye en su orden en las regiones Huetar Norte, Brunca, Huetar Caribe, Chorotega y Pacífico Central. 
Tabla 1. Costa Rica, zona rural: hogares y personas según región.

\begin{tabular}{lcc}
\hline & Hogares & Personas \\
\hline Zona rural & 418.935 & 1.354 .630 \\
Central & 128.529 & 420.978 \\
Chorotega & 55.658 & 169.166 \\
Pacífico Central & 31.321 & 99.021 \\
Brunca & 64.172 & 206.327 \\
Huetar Caribe & 61.579 & 192.959 \\
Huetar Norte & 77.676 & 266.179 \\
\hline
\end{tabular}

Elaboración propia con datos de la ENAHO 2017.

En cuanto a la composición de estos hogares según su tamaño, el porcentaje de hogares unipersonales es cercano a $13 \%$ y llega incluso a registrar porcentajes superiores en las regiones Chorotega, Brunca y Huetar Caribe. En promedio, los hogares rurales tienen de uno a tres miembros, similar al que se registra a nivel nacional. El porcentaje de hogares con más de tres miembros es cerca de $40 \%$, que resulta idéntico en todas las regiones. Por su parte, el porcentaje de hogares con jefatura femenina es de $30 \%$ y es superior en regiones como la Chorotega, Brunca y Huertar Caribe.

Tabla 2. Costa Rica, zona rural: composición de los hogares, según tamaño y porcentaje de hogares con jefatura femenina

\begin{tabular}{lcccc}
\hline & $\begin{array}{c}\text { Hogares } \\
\text { unipersonales } \\
(\%)\end{array}$ & $\begin{array}{c}\text { Hogares con más } \\
\text { de tres miembros } \\
(\%)\end{array}$ & $\begin{array}{c}\text { Promedio } \\
\text { miembros }\end{array}$ & $\begin{array}{c}\text { Hogares con } \\
\text { jefatura } \\
\text { femenina }\end{array}$ \\
\hline Zona rural & 12,7 & 39,9 & 3,23 & 30,3 \\
Central & 11,9 & 41,1 & 3,28 & 28,3 \\
Chorotega & 13,5 & 35,8 & 3,04 & 35,5 \\
Pacífico Central & 10,9 & 38,2 & 3,16 & 26,2 \\
Brunca & 14,3 & 39,1 & 3,22 & 33,7 \\
Huetar Caribe & 13,3 & 36,5 & 3,13 & 32,5 \\
Huetar Norte & 12,2 & 39,9 & 3,43 & 27,0 \\
\hline
\end{tabular}

Elaboración propia con datos de la ENAHO 2017. 
Con respecto al nivel de instrucción de la población rural, en su mayoría como máximo solo cuentan con sexto de primaria y un porcentaje muy reducido tiene bachillerato de secundaria. El porcentaje de personas con estudios universitarios o al menos que cuenten con una carrera técnica también resulta bajo: en ninguna región supera $10 \%$. Por su parte, el promedio de escolaridad es en todas las regiones es alrededor de 6 años.

Esta situación induce a la población rural a tomar trabajados no calificados o semicalificados con un rango salarial entre $9.822,07$ colones y $10.680,80 \mathrm{co}-$ lones por jornada ordinaria, según la escala de salarios mínimos del Ministerio de Trabajo y Seguridad Social (MTSS) del 2017. Además, se limita el acceso a los mercados laborales, principalmente si consideramos que las actividades económicas que secundan las del sector primario son los servicios y, con ello, el dominio de un segundo y tercer idioma.

Tabla 3. Costa Rica, zona rural: nivel de instrucción de la población

\begin{tabular}{lccccc}
\hline \multicolumn{1}{c}{ Regiones } & $\begin{array}{c}\text { Años } \\
\text { promedio } \\
\text { de } \\
\text { escolaridad }\end{array}$ & $\begin{array}{c}\text { Sin nivel } \\
\text { de } \\
\text { instrucció } \\
\mathbf{n}\end{array}$ & $\begin{array}{c}\text { Primaria } \\
\text { completa }\end{array}$ & $\begin{array}{c}\text { Secundaria } \\
\text { académica } \\
\text { completa }\end{array}$ & $\begin{array}{c}\text { Educación } \\
\text { superior de } \\
\text { pregrado y } \\
\text { grado }\end{array}$ \\
\hline Zona rural & 6,17 & 16,2 & 25,4 & 8,7 & 7,4 \\
Central & 6,50 & 13,6 & 28,0 & 9,3 & 9,5 \\
Chorotega & 6,60 & 13,6 & 23,5 & 11,2 & 8,4 \\
Pacífico Central & 6,46 & 14,8 & 24,9 & 8,3 & 8,7 \\
Brunca & 5,93 & 16,4 & 25,7 & 7,3 & 7,1 \\
Huetar Caribe & 5,76 & 17,7 & 26,2 & 8,1 & 5,0 \\
Huetar Norte & 5,75 & 21,3 & 22,0 & 7,7 & 5,0 \\
\hline
\end{tabular}

Elaboración propia con datos de la ENAHO 2017.

En cuanto al porcentaje de personas ocupadas de la zona rural, en todas las regiones se registran cifras muy similares: un poco más de $50 \%$ de la población rural se mantiene en alguna ocupación. La tasa de desempleo en todas las regiones rurales es relativamente baja, con cifras inferiores a $5 \%$. A nivel nacional, la ENAHO 2017 registra una tasa de desempleo de 7,5 ${ }^{1}$. Es importante enfatizar que hasta el 2013 el desempleo era mayor en la zona rural que en la urbana. Sin embargo, a partir de ese año los patrones de comportamiento cambiaron, en el sentido de que el desempleo rural continuó bajando pero el urbano creció (Loría y Martinez, 2016, 9). A propòsito de la población fuera de la fuerza de trabajo, es decir, los menores de 15 años, adultos mayores o personas con discapacidad, representa $45,6 \%$ de la población rural total.
1. Es importante aclarar que el dato sobre la tasa de desempleo que registra la ENAHO no es comparable con la que registra la Encuesta Continua de Empleo (ECE), debido a que metodológicamente son encuestas distintas. LA ECE se concentra en la medición de la participación laboral de las personas y la situación de empleo y desempleo en el corto plazo; surge en el 2010 , ante la inexistencia en el país de estadísticas laborales de corto plazo (Loría y Martínez, 2016, 4). 
Tabla 4. Costa Rica, zona rural: porcentaje de ocupados, desempleo abierto, fuera de la fuerza de trabajo

\begin{tabular}{lccc}
\hline \multicolumn{1}{c}{ Regiones } & Ocupados & $\begin{array}{c}\text { Desempleado } \\
\text { abierto }\end{array}$ & $\begin{array}{c}\text { Fuera de la fuerza } \\
\text { de trabajo }\end{array}$ \\
\hline Zona rural & 50,3 & 4,0 & 45,6 \\
Central & 53,2 & 4,0 & 42,8 \\
Chorotega & 48,6 & 3,9 & 47,6 \\
Pacífico Central & 50,4 & 2,5 & 47,1 \\
Brunca & 48,5 & 3,8 & 47,8 \\
Huetar Caribe & 50,4 & 4,3 & 45,3 \\
Huetar Norte & 48,1 & 4,7 & 47,1 \\
\hline
\end{tabular}

Elaboración propia con datos de la ENAHO 2017.

A pesar de que la tasa de desempleo en las zonas rurales es baja, como se mencionó anteriormente, los bajos niveles de escolaridad que muestra la población rural influye de manera directa en el tipo y la calidad de empleo por el que pueden optar. En este contexto, el sector informal provee un conjunto de unidades de producción de bienes o prestación de servicios, que funcionan en pequeña escala, con una organización rudimentaria, y escasa división entre trabajo y capital; adicionalmente, las relaciones de empleo (cuando existen) no están amparadas por contratos formales que supongan garantías y derechos.

Durante la Decimoquinta Conferencia Internacional de Estadísticos del Trabajo en 1993, se definió el sector informal de trabajo haciendo referencia principalmente a las características de la empresa o negocio como unidad económica de estudio. En concordancia con el marco del Sistema de Cuentas Nacionales de las Naciones Unidades (SCN, 1993), dichos establecimientos forman parte de las empresas comerciales de los hogares no constituidas en sociedad. En este sentido, el sector informal debe de verse como un conjunto de unidades de producción de bienes o prestación de servicios, que funcionan en pequeña escala, con una organización rudimentaria, escasa división entre trabajo y capital; además, las relaciones de empleo (cuando existen) no están amparadas por contratos formales que supongan garantías y derechos (Delgado, 2013, 37).

En efecto, de acuerdo con los datos, cerca de $40 \%$ de la población rural con trabajo se dedica a ocupaciones consideradas como elementales (no calificadas), ya sea como asalariados de empresas privadas e instituciones públicas, o en un negocio, una empresa o actividad propia. 
Tabla 5. Costa Rica, zona rural: porcentaje de la población en ocupaciones elementales y categoría en el empleo

\begin{tabular}{|c|c|c|c|}
\hline \multirow[b]{2}{*}{ Regiones } & \multirow{2}{*}{$\begin{array}{c}\begin{array}{c}\text { Ocupación } \\
\text { principal }\end{array} \\
\begin{array}{l}\text { En ocupaciones } \\
\text { elementales (\%) }\end{array}\end{array}$} & \multicolumn{2}{|c|}{ Categoría en el empleo } \\
\hline & & $\begin{array}{l}\text { Como empleado para un } \\
\text { patrón, empresa o } \\
\text { institución (\%) }\end{array}$ & $\begin{array}{l}\text { Un negocio, empresa } \\
\text { o actividad propia (\%) }\end{array}$ \\
\hline Zona rural & 40,0 & 74,2 & 22,2 \\
\hline Central & 32,5 & 75,0 & 22,2 \\
\hline Chorotega & 42,8 & 71,1 & 24,0 \\
\hline Pacífico Central & 37,0 & 78,9 & 18,6 \\
\hline Brunca & 43,3 & 70,8 & 25,9 \\
\hline Huetar Caribe & 48,1 & 78,7 & 17,8 \\
\hline Huetar Norte & 47,7 & 72,3 & 22,8 \\
\hline
\end{tabular}

Elaboración propia con datos de la ENAHO 2017.

En términos generales, se tratan de ocupacione relacionadas con labores agrícolas, trabajo doméstico, construcción y ventas al por mayor y menor en algún establecimiento comercial. En regiones con una alta vocación agrícola como la Brunca, la Huetar Caribe y la Huetar Caribe, resulta lógico que un bajo porcentaje de la población rural se encuentre vinculada al sector de servicios.

Tabla 6. Costa Rica, zona rural: porcentaje de población en ocupaciones elementales según rama de actividad principal

\begin{tabular}{lccccc}
\hline Regiones & $\begin{array}{c}\text { Agricultura } \\
\text { (A) }\end{array}$ & $\begin{array}{c}\text { Hogares } \\
\text { como } \\
\text { empleadores } \\
\text { (B) }\end{array}$ & $\begin{array}{c}\text { Construcci } \\
\text { ón (C) }\end{array}$ & $\begin{array}{c}\text { Comercio al } \\
\text { por mayor y al } \\
\text { por menor (D) }\end{array}$ & A+B+C+D \\
\hline Zona rural & 44,4 & 19,2 & 11,0 & 4,2 & 78,8 \\
Central & 33,4 & 26,2 & 12,4 & 4,8 & 76,8 \\
Chorotega & 25,7 & 30,3 & 10,4 & 3,6 & 70,0 \\
Pacífico Central & 29,3 & 19,7 & 15,1 & 2,7 & 66,8 \\
Brunca & 48,0 & 15,9 & 10,7 & 2,2 & 76,8 \\
Huetar Caribe & 63,5 & 9,7 & 8,3 & 4,7 & 86,2 \\
Huetar Norte & 59,0 & 11,9 & 10,7 & 5,5 & 87,1 \\
\hline
\end{tabular}

Elaboración propia en base a datos de INEC. Encuesta Nacional de Hogares (ENAHO 2017). 
De esta manera, existe una conformación diversa de grupos familiares que requieren un ingreso estable para subsistir sin la formación necesaria para desempeñar tareas calificadas. Se trata únicamente ocupaciones elementales o no calificadas, las cuales implican según la Clasificación de Ocupaciones de Costa Rica (INEC, 2014), la realización de tareas sencillas y rutinarias que pueden requerir la utilización de herramientas manuales y un esfuerzo físico considerable, como por ejemplo limpiar, tareas básicas de mantenimiento, de preparación de alimentos, tareas simples en la agricultura, la construcción y fabricación de productos, e incluso la clasificación de productos, embalar y desembalar productos a mano y llenar estanterías o proporcionar diversos servicios en la calle, etc.

Si se examinan los salarios monetarios brutos que perciben estos trabajadores en todas las regiones, los más altos se registran la construcción y el comercio. Les siguen los trabajadores de la agricultura: se notan algunas diferencias entre regiones, en particular la región Huetar Caribe y la Pacífico Central con las restantes. Por su parte, los salarios más bajos son registrados por los trabajadores que realizan labores domésticas o de mantenimiento en los hogares.

Tabla 7. Costa Rica, zona rural: salario monetario bruto promedio de los trabajadores no calificados según rama de actividad en el empleo principal

\begin{tabular}{lcccc}
\hline \multicolumn{1}{c}{ Regiones } & Agricultura & $\begin{array}{c}\text { Hogares como } \\
\text { empleadores }\end{array}$ & Construcción & $\begin{array}{c}\text { Comercio al } \\
\text { por mayor y al } \\
\text { por menor }\end{array}$ \\
\hline Zona rural & 210.748 & 127.263 & 232.274 & 251.638 \\
Central & 181.077 & 136.931 & 219.748 & 227.601 \\
Chorotega & 216.387 & 137.658 & 277.560 & 271.931 \\
Pacifico Central & 212.518 & 130.759 & 210.504 & 268.244 \\
Brunca & 136.749 & 93.481 & 257.249 & 249.912 \\
Huetar Caribe & 257.860 & 102.045 & 210.715 & 224.360 \\
Huetar Norte & 233.685 & 130.223 & 235.052 & 282.094 \\
\hline
\end{tabular}

Elaboración propia con datos de la ENAHO 2017.

Estas diferencias que se establecen entre los salarios de los trabajadores se explica en razón de la cantidad de horas trabajadas por semana. Es decir, los trabajadores de la construcción y la agricultura tienen los salarios promedios más altos, pero también son los que más horas trabajan. En el caso de los trabajadores domésticos y de mantenimiento de hogares, cuentan con el salario promedio más bajo y la menor cantidad de horas trabajadas, lo cual indica que este grupo de trabajadores, en su mayoría, se contrata por horas o por jornadas de medio tiempo. 
Tabla 8. Costa Rica, zona rural: horas normales semanales promedio laboradas. Trabajadores no calificados según rama de actividad en el empleo principal

\begin{tabular}{lcccc}
\hline \multicolumn{1}{c}{ Regiones } & Agricultura & $\begin{array}{c}\text { Hogares como } \\
\text { empleadores }\end{array}$ & Construcción & $\begin{array}{c}\text { Comercio al } \\
\text { por mayor } \mathbf{y} \\
\text { al por menor }\end{array}$ \\
\hline Zona rural & 37,7 & 25,7 & 36,8 & 33,4 \\
Central & 35,4 & 26,3 & 45,8 & 34,8 \\
Chorotega & 40,5 & 27,0 & 44,0 & 30,2 \\
Pacífico Central & 35,0 & 26,6 & 46,0 & 39,2 \\
Brunca & 29,9 & 22,6 & 41,6 & 39,4 \\
Huetar Caribe & 47,3 & 22,2 & 43,8 & 36,8 \\
Huetar Norte & 44,4 & 26,9 & 34,7 & 48,4 \\
\hline
\end{tabular}

Elaboración propia con datos de la ENAHO 2017.

En cuanto a los aportes a la seguridad social, un poco más de la cuarta parte cotiza. No obstante, en su condición de asalariados, este porcentaje de trabajadores que cotizan debería ser muy superior. Esto no implica que se encuentran del todo descubiertos por el seguro social (atención médica) porque lo pueden tener por otros medios distintos a su condición de asalariado, ya sea por el Estado, gracias a un familiar de asegurado directo, por pago voluntario, como trabajador independiente o por convenio, como sucede con los trabajadores agrícolas que son parte de organizaciones como la Unión de Productores Independientes y Actividades Varias (UPIAV) o la Unión Nacional de Pequeños y Medianos Productores Agropecuarios (UPA Nacional).

Tabla 9. Costa Rica, zona rural: distribución porcentual de la forma en que adquieren el seguro social los trabajadores no calificados

\begin{tabular}{lcccccc}
\hline \multicolumn{1}{c}{ Regiones } & $\begin{array}{c}\text { No } \\
\text { asegurad } \\
\text { o }\end{array}$ & $\begin{array}{c}\text { Asalaria } \\
\text { do }\end{array}$ & $\begin{array}{c}\text { Por el } \\
\text { Estado }\end{array}$ & $\begin{array}{c}\text { Familiar } \\
\text { de } \\
\text { asegurado } \\
\text { directo }\end{array}$ & $\begin{array}{c}\text { Voluntari } \\
\text { o }\end{array}$ & $\begin{array}{c}\text { Otras } \\
\text { formas de } \\
\text { seguro }\end{array}$ \\
\hline Zona rural & 28,1 & 33,8 & 7,2 & 11,1 & 12,6 & 7,2 \\
Central & 24,1 & 30,1 & 5,3 & 14,4 & 13,9 & 12,2 \\
Chorotega & 25,6 & 40,3 & 8,8 & 8,6 & 9,9 & 6,8 \\
Pacífico Central & 27,7 & 43,6 & 6,0 & 5,7 & 8,9 & 8,1 \\
Brunca & 28,9 & 23,0 & 10,5 & 8,8 & 20,2 & 8,6 \\
Huetar Caribe & 20,7 & 54,4 & 7,6 & 6,8 & 5,6 & 4,9 \\
Huetar Norte & 33,2 & 39,9 & 4,4 & 7,7 & 12,0 & 2,8 \\
\hline
\end{tabular}

Elaboración propia con datos de la ENAHO 2017.

Incluso con estas consideraciones, un poco más de la cuarta parte no tienen seguro social en absoluto. La cifra más alta y quizás preocupante se halla en la región Huetar Caribe con $33,2 \%$ de los trabajadores calificados sin seguro social. La situación es distinta en la región Huetar Caribe, donde 54,4\% de 
los trabajadores asalariados tienen seguro social; esto se debe, principalmente, a la presencia de las grandes empresas transnacionales bananeras que tratan de cumplir sus obligaciones con la seguridad social.

La principal preocupación con respecto a que los trabajadores no coticen a la seguridad social como asalariados, es que en el futuro no tendrán acceso a las pensiones del régimen contributivo, lo cual agravaría su condición de pobreza y comprometería al Estado a proporcionar una pensión bajo el régimen no contributivo. Tal situación también implica que muchos empleadores no le están haciendo frente a sus obligaciones de seguridad social.

Si se examina la condición de migrante de la población rural, sobre todo la migración interna, se observa que una cuarta parte de las personas ha tenido que migrar de su región de nacimiento en búsqueda de oportunidades laborales o mejor acceso a los servicios.

Tabla 10. Costa Rica, zona rural: condición de migrante de la población rural

\begin{tabular}{lccc}
\hline \multicolumn{1}{c}{ Regiones } & No migrante & Migrante interno & Migrante externo \\
\hline Zona rural & 65,9 & 25,6 & 8,5 \\
Central & 72,7 & 22,2 & 5,1 \\
Chorotega & 71,6 & 20,9 & 7,5 \\
Pacífico Central & 61,0 & 29,1 & 9,9 \\
Brunca & 67,9 & 28,5 & 3,6 \\
Huetar Caribe & 57,1 & 33,4 & 9,5 \\
Huetar Norte & 58,4 & 24,9 & 16,7 \\
\hline
\end{tabular}

Elaboración propia con datos de la ENAHO 2017.

En este contexto, se visualiza un patrón estructural de desigualdad e impedimento práctico real para la búsqueda de mejores opciones y condiciones laborales: las posibilidades de conseguir empleo se limitan a la dinámica productiva de la región. Las personas que deseen abrir sus expectativas educativas y laborales mediante la dinámica de la movilidad interna tienen que considerar la posibilidad de no retorno o un retorno condicionado por las expectativas laborales reales y las de desarrollo de capacidades.

Todo lo anterior viene a redefinir la posición de la población rural en términos de generación de ingresos. En efecto, si se examina la información de la ENAHO 2017 referente a los quintiles de ingreso per cápita, 60\% se ubica en los dos quintiles más bajos; en las regiones Brunca y la Huetar Norte, es alrededor de $70 \%$. En el caso de la primera región, $44,4 \%$ de la población se ubica en el quintil de ingresos más bajo. 
Tabla 11. Costa Rica, zona rural: porcentaje de los hogares en los quintiles I y II de ingreso per cápita y condición de pobreza

\begin{tabular}{lcccc}
\hline Regiones & $\begin{array}{c}\text { Quintil I de } \\
\text { ingresos }\end{array}$ & $\begin{array}{c}\text { Quintil II de } \\
\text { ingresos }\end{array}$ & $\begin{array}{c}\text { Pobreza } \\
\text { extrema }\end{array}$ & $\begin{array}{c}\text { Pobreza no } \\
\text { extrema }\end{array}$ \\
\hline Zona rural & 31,6 & 24,4 & 7,9 & 16,2 \\
Central & 25,6 & 23,7 & 5,9 & 12,8 \\
Chorotega & 31,0 & 24,6 & 5,4 & 17,6 \\
Pacífico Central & 28,2 & 23,0 & 8,8 & 14,2 \\
Brunca & 44,4 & 23,5 & 12,4 & 20,0 \\
Huetar Caribe & 29,2 & 28,0 & 6,5 & 16,3 \\
Huetar Norte & 37,7 & 23,7 & 10,0 & 18,5 \\
\hline
\end{tabular}

Elaboración propia con datos de la ENAHO 2017.

En términos del indicador de pobreza, cerca de $25 \%$ de los hogares rurales se encuentran en una situación de pobreza extrema o no extrema; muy superior al porcentaje nacional, que se ubica alrededor de $20 \%$. La región Brunca registra el mayor porcentaje de hogares en condiciones de pobreza: $32,4 \%$. En la región Pacífico Central, el porcentaje de personas en condiciones de pobreza es menor que el anterior, pero en todo caso, superior a la cifra nacional de $20 \%$.

Es evidente que la población instalada en los territorios rurales podrá mejorar sus condiciones por medio de cambios en la inversión, la estructura del empleo local y el funcionamiento del sistema institucional para el acondicionamiento y la implementación de políticas públicas y estrategias de atracción y generación de nuevos mercados laborales. Para ello, es necesario apuntalar, con claridad operativa y contextualizada, las estrategias de desarrollo diferenciado de la política económica nacional; y, por consiguiente, la elaboración de directrices estratégicas que relacionen la promoción de empleo con el contexto, lo mismo que vincular las condiciones y necesidades locales con el rumbo de la economía.

\section{Rumbo económico, empleo y desarrollo rural}

Entre las alternativas que se plantean para revertir la situación de pobreza y desigualdad, se encuentran aquellas dirigidas al fortalecimiento de la producción y a la diversificación del trabajo, así como las políticas de fomento de la investigación, la extensión y la innovación tecnológica para mejorar los salarios, el acceso a la salud y la educación y la disponibilidad y el acceso a alimentos.

El ideal de "progreso", producto de la revolución industrial y, posteriormente, de la revolución tecnológica, tendió a mimetizar las contradicciones globales del sistema capitalista, reivindicando el método clásico de crecimiento lineal, por etapas, cuantificable y predecible. En este sentido, la consolidación del capital empresarial-privado y los ajustes estructurales que promovieron la 
modernización y nueva industria, redujeron sustancialmente las posibilidades de un desarrollo a "escala humana". Por el contrario, afianzaron un modelo dual funcionalista en que el beneficio mutuo y la dependencia del centro con respecto a su periferia atascaron el intento de lograr un desarrollo endógeno que fuera contrario a la idea sistema del mundo.

De esta forma, los debates ideológicos se avivaban cuando los defensores del llamado "desarrollo hacia afuera" y la perspectiva de la acumulación de capital y de la dominación política certificaban verdaderas prolongaciones de los territorios extranjeros, mientras que, por otro lado, los aportes de la teoría marxista de la dependencia analizaban el desplazamiento del sistema oligárquico-terrateniente a uno centrado en las necesidades y variables regionales; esto implica innovación permanente focalizada en la calidad del producto (Rivera, 2015,19).

Así, las relaciones entre los actores sociales con los medios de producción que acentuaban el trabajo de oficio y cuyo patrono tenía el control absoluto del trabajo, han cambiado paulatinamente. Además, la desterritorialización del trabajo como "la venta a domicilio, que subvierten los conceptos de jornada de trabajo y de espacio productivo" (De la Garza, 2001) luchan por la apertura de nuevas fuentes de empleo y no por la reinstalación de puesto de trabajo de oficio.

En el caso particular de Costa Rica, las reformas estructurales aplicadas a partir de la década de los ochenta orientadas a la liberación económica, la desregulación y la privatización de los sectores productivos y algunos servicios para favorecer los intercambios comerciales y la atracción de inversión extranjera directa, transformaron la estructura de empleo y las actividades económicas en el medio local y rural, donde no se logra fortalecer el empleo de calidad y los mercados de trabajo en los territorios periféricos. En consecuencia se consolidan la concentración de las actividades económicas en el Gran Área Metropolitana (GAM) y el aumento de los costes de la producción; $y$, con ello, la localización de empresas en las regiones centrales, el desarrollo de la infraestructura económica y los servicios, la calidad de la educación y la dotación de recursos humanos calificados, etc.

Precisamente, el fundamento que determina -en gran medida- las desigualdades en los niveles de desarrollo de las poblaciones urbanas con respecto a las poblaciones rurales se basa en el análisis histórico de la producción agropecuaria y la generación de materias primas producto de los modelos de desarrollo ejecutados a finales del siglo XIX y principios del siglo XX. Por eso, ha existido la acumulación de un capital latifundista que acaparó las cúpulas políticas y las grandes obras de infraestructura, altiempo que ha segmentado los territorios.

Este proceso impulsó una legislación que permitió, por un lado, el desarrollo empresarial junto con la inversión del Estado para construir las bases tecnológicas y burocráticas con las que contamos actualmente; y, por otro, la creación de garantías sociales para salvaguardar la salud, la educación y el trabajo en condiciones adecuadas. Sin embargo, el reacomodo de los compro- 
misos adquiridos en esa primera fase de desarrollo agropecuario latifundista por parte de la clase política, trajo consigo el asentamiento de niveles de especialización del trabajo relacionados con la producción de las materias, su industrialización y comercialización, en una especie de fordismo que consolidó las diferencias estructurales de principios del siglo $X X$ en desigualdades sociales que involucraban un ascetismo laboral y, por ende, una baja en el ingreso familiar rural.

Desde la configuración y promoción del Código de Trabajo de 1943, producto de la coyuntura política asociada a la promulgación de las garantías sociales y el desarrollo de un Estado de bienestar caracterizado por la regulación estatal en el ámbito económico y la unión -a la fuerza- de la industria con la producción agropecuaria, se permitió aumentar la productividad. Pero hubo un estancamiento de las condiciones laborales y se inició de una brecha de ingresos y dependencia económica.

En este sentido, la inserción a los mercados internacionales de un país cuya base productiva es la manufactura y los servicios, tuvo un fuerte impulso en los noventa con los Programas de Ajuste Estructural (PAE) con políticas tendientes a lograr el equilibrio fiscal con nuevas tarifas arancelarias. De esta forma, se programaron tres fases (Arias y Muñoz, 2007, 9): la estabilización que prepara la economía para el cambio de sus principales parámetros macroeconómicos; el ajuste estructural que incluye modificaciones en los precios relativos, la orientación de la estrategia de desarrollo hacia la apertura, la liberalización comercial, la eficiencia y la productividad y la transformación estructural que refiere a cambios mayores y de carácter estratégico como los relacionados con el cambio tecnológico, la calificación y calidad de la mano de obra, el desarrollo sostenido, la equidad y la competitividad de largo plazo. Estos cambios, en esencia, no modificaron la estructura productiva costarricense basada en la exportación de materias primas e importación de bienes manufacturados; de ahí que los salarios aumentan, pero en desequilibrio con la inflación y el costo de la vida.

Al menos en el 2017, el Consejo Nacional de Salarios aumento en $2,43 \%$ el salario mínimo para el sector privado, que conjunta $80 \%$ de la fuerza laboral del país. Empero, las proyecciones del Banco Central de Costa Rica (BCCR) estiman una inflación de 4\% para el 2018. Asimismo, el Pacto Mundial para el Empleo (2009) y la Declaración de Lima (2014) acordaron paquetes de estímulo macroeconómico, la formalización de la economía, el impulso al empleo juvenil y al empleo verde ${ }^{2}$; todos son aún retos que se tienden a desvanecer en medio de la crisis fiscal ${ }^{3}$. En este sentido, el factor determinante del desarrollo de un país con un modo de producción sustentable es la correlación entre la búsqueda de un nuevo potencial redistributivo del trabajo en clave territorial -o espacio local jurídicamente diferenciado 4 - y los grados de interrelación entre la producción, la oferta y la demanda de los mercados y el Estado.
2. Empleos verdes, según la OIT, son empleos decentes que contribuyen a preservar y restaurar el medio ambiente ya sea en los sectores tradicionales como la manufactura o la construcción o en nuevos sectores emergentes como las energías renovables y la eficiencia energética.

http://www.ilo.org/global/topi cs/green-jobs/news/WCMS_325253 /lang--es/index.htm

3. Los gastos del Gobierno de Costa Rica han superado en $6,2 \%$ el Producto Interno Bruto (PIB). Supone una

desaceleración de la actividad económica y un incremento de los gastos, lo que conlleva a un desfase en la recaudación con respecto a la estructura productiva actual.

4. Antonio Carlos Wolkmer establece que la participación, el control y la representación vinculante de los intereses de todos los sectores de la sociedad, en nivel local, ya sea sobre la experiencia de sujetos individuales o sobre la expresión de sujetos colectivos (Wolkmer, 2002, 139), debe contemplar el espacio público comunitario. 


\section{El reposicionamiento de los territorios rurales}

Es innegable que la creciente interdependencia económica y alta competitividad tiene como juicio de hecho dos vertientes ineludibles que determinan en gran medida el campo de acción del trabajo por el cual se van a distribuir los nuevos puestos de trabajo. La primera vertiente es el grado de inserción en los mercados nacional e internacional y el uso de las tecnologías para la especialización del trabajo, como instrumento básico para impulsar y aumentar las transformaciones productivas. La otra vertiente es la acción intergubernamental para comprender los flujos transfronterizos y de movilidad social, que implica la creación de imaginarios sociales sobre la persona que se traslada y cambia su asentamiento en razón de diversas condiciones estructurales que determinan la decisión, personal y familiar, de adoptar un nuevo rumbo en su situación de vida.

Estas dos vertientes insertas en el factor determinante del desarrollo laboral en territorios rurales se pueden esquematizar de la siguiente forma:

Figura 1

Territorios rurales

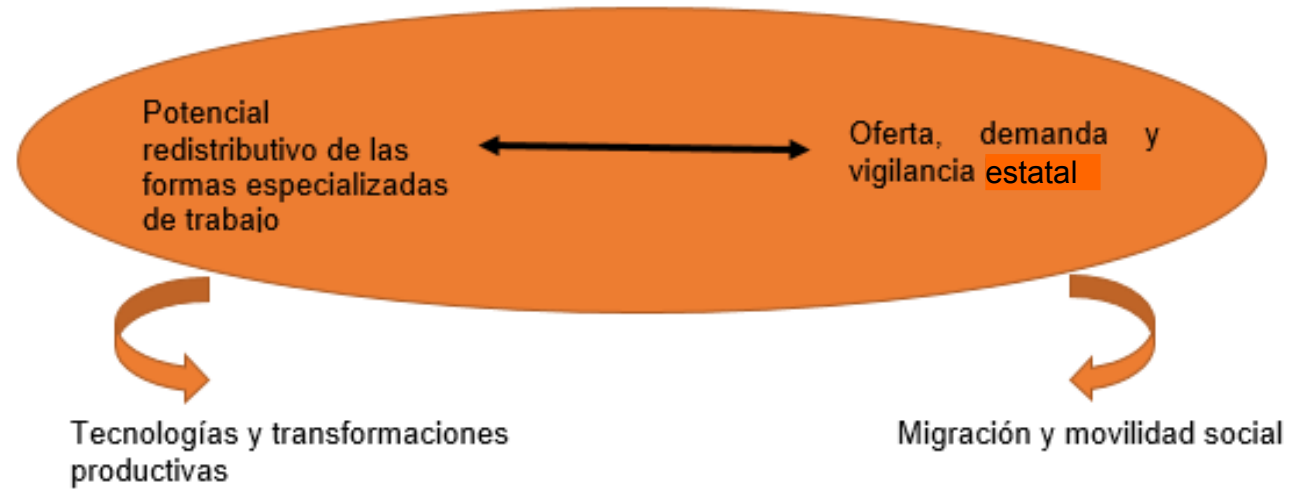

Fuente: Elaboración propia, 2017

Al menos en su acepción básica, la territorialidad como parte de los ejes de funcionamiento del Estado-nación se asienta sobre la base de una cartografía simbólica, la cual a su vez legitima el espacio de tierra demarcado con discursos, paisajes, iconografías y costumbres en esquemas jurídicos que promueven una identidad nacional, al mismo tiempo que tensiona la eficacia y relevancia normativa que el paradigma de la globalización -de la libertad de frontera- prolifera.

Así, el territorio es considerado como un espacio físico orgánico, rizomático y flexible, donde convergen formas del trabajo específicas, producto de las particularidades -ambientales, culturales, políticas y jurídicas- cuyo asidero histó- 
rico determina, desde la lógica instrumental capitalista, el valor o potencialidad para el desarrollo. Este sesgo es el principal problema estructural que permea el mundo del trabajo, particularmente el desempleo juvenil, en los territorios rurales $\mathrm{y}$, por consiguiente, todas sus relaciones.

El nuevo potencial redistributivo de las formas especializadas de trabajo emergentes, generadoras de ingreso, así como las no necesariamente remuneradas -exclusivas de cierto tipo de trabajo- en el corolario de un plan de desarrollo o política social, debe interpelar a la cohesión social y la generación de capacidades comunitarias. Con este ligamen se forma, por la codependencia de los actores en la toma de decisiones, una coordinación que escalonadamente genera distintos nichos de actividades productivas $\mathrm{y}$, de ese modo, una especialización de actividades laborales útiles para el desarrollo general del territorio.

Esta dinámica es la interacción entre la dispersión geográfica y nuevos tipos de economías de aglomeración. La especificación de un marco analítico común para estas dos muy diversas formas espaciales deberían permitirnos, en primer lugar, desarrollar una aproximación más aguda sobre las políticas; y, en segundo lugar, establecer el poder de negociación actual de los actores urbano/regionales, así como nuevos tipos de actores inter-gubernamentales (Sassen, 2007 (a), 10).

Para que este nuevo potencial redistributivo se constituya, es fundamental comprender que la población económicamente activa está compuesta por procesos de movilidad social o migración laboral que son producto de un continuum colonial que traza la salida y llegada de migrantes, quienes deciden encontrar mejores condiciones y formar espacios transnacionales cuyo génesis -sin que ello determine una causalidad absoluta- se encuentra en "los lazos entre las antiguas colonias y los países colonizadores" (Sassen, 2007 (b), 169).

Tal impulso, abonado a la apertura o diversidad de puestos de trabajo, establece una vía de fuentes de ingresos familiares. Pero, a su vez, también se constituye en una actividad de no retorno del territorio rural. Esta ubicación da pista para valorar el trabajo en términos morales e identitarios. Es decir, con una decisión que interpela otros factores emocionales y afectivos, de paz, estabilidad; y no solo criterios económicos de cantidad de salario, así como la codependencia de la persona trabajadora (sujeto), la organización del trabajo (empresa, cooperativa, gubernamental) y el modelo o esquema de desarrollo (política). Todo lo anterior estaría en función de erradicar la pobreza y establecer mejores condiciones de vida para la población.

De esta manera, la concepción ampliada del trabajo permite comprender la complejidad de los procesos de cambio que se desarrollan desde el proceso productivo, la reorganización social del trabajo, sus colectivos y tendencias, la incidencia la globalización en las formas capitalistas de producción, y la dependencia y creación de nuevos los puestos de trabajo y competencias a partir del desarrollo de las tecnologías. De ese modo, se permite resignificar el mundo del trabajo como instancia conformadora de subjetividades e identi- 
dades, cuyas transformaciones productivas, historicidad de los colectivos y tendencias de los modelos de desarrollo se involucran y reaccionan en un juego integrador en la personalidad del trabajador y en los "los efectos de la condición de la vida" (Offe, 1985). Es ineluctablemente necesario comprender lo anterior en referencia a los territorios rurales, ya que su acción ciudadana o promoción de estrategias de desarrollo están vinculadas con un arraigo territorial que forma, en perspectiva, su personalidad, sus intereses y sus decisiones políticas.

\section{Conclusiones}

Resulta imperioso integrar los aspectos propios de una estrategia de empleo para el desarrollo rural relacionados con el salario y las horas pagadas, la seguridad social, el marco institucional regulatorio, la movilidad nacional e internacional del empleo y la circulación de los trabajadores. Es preciso analizar cómo el país regula la libertad de circulación de los trabajadores, la prestación de los servicios y las acciones del empresariado internacional.

El impulso al empleo en territorios rurales y la apropiación de los medios de producción por parte de los trabajadores requiere una estrategia cuya política pública no sea espacial. Por lo tanto, es necesario plantear aspectos clave para una política de empleo local y rural; por ejemplo, las políticas y las estrategias de desarrollo local posibles que dinamicen los mercados locales y rurales de trabajo; los emprendimientos, la innovación y la capacidad de inserción de trabajadores profesionales a nivel local y rural; la transición de los trabajadores hacia la apropiación de los medios de producción y la oportunidad para los sectores excluidos, en condición de desigualdad del modelo económico (trabajadores de cuenta propia, jóvenes, mujeres y adultos).

El estudio de lo anterior permitirá elaborar directrices para fortalecer el empleo en territorios rurales tomando en cuenta el contexto, la vinculación de las políticas públicas sociales y económicas y las estrategias de dinamización de los mercados locales, empleos verdes, la atracción de la inversión extranjera, la movilidad laboral, el marco macroeconómico, la política social y la realidad política del país. Para ello, es necesario tener en consideración una concepción ampliada del trabajo y su incidencia en el análisis de la situación actual del empleo. En este sentido, el trabajo desde la óptica clásica entiende la acción instrumental como la simple relación obrero patronal, como valoración racional de la efectividad del trabajo en la regulación de la vida en sociedad. Esta crítica impulsa y vuelve necesaria una relectura del mundo del trabajo y de su potencialidad emancipadora como proyecto social en una transformación o amplitud de la relación tradicional oferta-demanda, con el fin de abrirla como una posible experiencia heterogénea.

En este sentido, surge la necesidad de redimensionar el espacio público como un espacio comunitario organizado y legalizado de manera diferencia$\mathrm{da}$, donde las posibilidades de crear segmentos asociativos -productivos, educativos y artísticos- no tengan que recurrir, exclusivamente, a la lógica po- 
lítico-deductiva gubernamental. La capacidad de crear (socialmente) posibilidades de desarrollo emergentes que coloquen en evidencia la interconexión e integración de los grupos humanos como sectores diferenciados y diferentes, es una tarea que debe ser enmarcada como prioritaria en el debate político-jurídico actual, más aún si se tiene en cuenta la pluralidad de interacciones jurídicas, económicas y discursivas que enmarcan la complejidad del desarrollo comunitario.

Por eso, la visualización de nuevos sujetos (y racionalidades) sociales que de modo permanente crean y recrean intereses y valores comunes, fortalecen nuestra expectativa acerca de las múltiples posibilidades que el desarrollo comunitario ejerce sobre la política nacional y regional, como una muestra más de que tanto el espacio local como el espacio global deben pensarse recursivamente.

\section{Bibliografía}

Arias Ramírez, Rafael y Muñoz López, Juan José. 2007. «La reforma económica y su impacto social en costa rica durante el período de ajuste estructural: apuntes críticos para el análisis». Revista Economía y Sociedad 31-32: 5-34. Acceso el 03 de mayo de 2018. http://revistas.una.ac.cr/index.php/economia/article/viewFile/172/131

De la Garza, Enrique. 2001. El futuro del trabajo - el trabajo del futuro. Buenos Aires. CLACSO, 2003.

Delgado Jiménez, Francisco. 2013. «El empleo informal en Costa Rica: Características de los ocupados y sus puestos de trabajo». Revista Ciencias Económicas 31(2): 35-51. Acceso el 01 de octubre de 2017. https://revistas.ucr.ac.cr/index.php/economicas/article/view/12694/1198 $\underline{2}$

INEC. 2001. «Conceptos y definiciones». Acceso el 18 de octubre de 2017. http://sistemas.inec.cr:8080/redatam/RpHELP/ENC.HOGARES/ENC.H OG_2001/Conceptos/Conceptos_y_definiciones.pdf

INEC. 2014. «Clasificación de Ocupaciones de Costa Rica 2011». Acceso el 20 de junio de 2017. http://www.inec.go.cr/sites/default/files/documentos/inec_institucional/m etodologias/documentos_metodologicos/38_metodologia_ocupaciones _2011.pdf

INEC. 2017 (a). «Encuesta Nacional de Hogares Julio 2017. Resultados generales». Acceso el 05 de mayo de 2018. http://www.inec.go.cr/sites/default/files/documetos-bibliotecavirtual/reenaho2017.pdf 
INEC. 2017 (b). «Encuesta Nacional de Hogares (ENAHO) 2017». San José. INEC, 2017.

Loría Sagot, Miguel y Martínez Castillo, Josué. 2016. «El desempleo en Costa Rica: evolución reciente y principales características». Serie Visión Costa Rica. Acceso el 05 de mayo de 2018. https://www.academiaca.or.cr/wp-content/uploads/2017/02/Desempleoen-Costa-Rica.pdf

Offe C. 1985. Disorganised Capitalism. Cambidge, Policy Press, 1985.

Rivera Alfaro, Ronald. 2015. «La interdisciplinariedad en las Ciencias Sociales». Revista Reflexiones 94 (1): 11-22. Acceso el 25 de agosto de 2017. https://revistas.ucr.ac.cr/index.php/reflexiones/article/view/20871/21013

Sassen, Saskia. 2007 (a). «El reposicionamiento de las ciudades y regiones urbanas en una economía global: ampliando las opciones de políticas y gobernanza». Revista eure (32,100): 9-34. Acceso el 25 de agosto de 2017. https://www.eure.cl/index.php/eure/article/view/1366/459

Sassen, Saskia. 2007 (b). Una sociología de la globalización. Buenos Aires. Katz Editores, 2007.

SCN. 1993. Sistema de cuentas nacionales. Banco Mundial

Wolkmer, Antonio Carlos. 2002. «Sociedad civil, poder comunitario y acceso democrático a La justicia». Pluralismo jurídico y alternatividad judicial. El otro derecho. Bogotá. ILSA-Instituto Latinoamericano de Servicios Legales Alternativos, 2002. 